\title{
Red de co-palabras en la bibliometría mexicana
}

\author{
Cristina Restrepo Arango* \\ Rubén Urbizagástegui Alvarado**
}

Se realizó un análisis de co-palabras utilizando las palabras clave en los documentos publicados sobre las metrías (bibliometría, informetría, cienciometría, patentometría, etc.) en México desde la década de 1970 hasta diciembre de 2012. Se usó el análisis de co-ocurrencia de palabras, la agrupación jerárquica y el mapa de la red social. Estas técnicas se aplicaron a 681 palabras clave (PC) con 1914 ocurrencias contenidas en 459 artículos. Se encontró que las palabras clave con la mayor ocurrencia son "Bibliometría" (10.6 \%), "México" (6.3\%), "Producción científica" (3\%), "América Latina" (3\%), "Factor de impacto" (1.8\%), "Indicadores bibliométricos" (1.8\%) y "Cienciometría" (1.7\%). La

* Pontificia Universidad Javeriana, Bogotá, Colombia. crestepoarango@gmail.com

** Universidad de California, EUA. ruben@ucr.com 
PC con el mayor grado de centralidad e intermediación de la red es "Bibliometría", mientras que la que posee el mayor grado de cercanía es "Evaluación de la investigación". También se encontraron cinco grupos de palabras clave que se comportan como cliques en la red.

Palabras clave: Bibliometría; Informetría; Cienciometría; México.

\section{Co-words network in Mexican Bibliometrics}

Cristina Restrepo-Arango and Rubén Urbizagástegui-Alvarado

\section{Abstract}

This work performs a co-words analysis using the keywords in the documents published on Bibliometrics, Informetrics, Sciencometrics, patent metrics, etc. in Mexico from the early 1970s until December 2012. The approach involves analysis of co-occurrence words, hierarchical clustering and the social network mapping of 681 keywords (KW) that found 1,914 occurrences in 459 papers. Results show the keywords with the highest occurrence are "Bibliometrics" (10.6\%), "Mexico" (6.3\%), "Scientific Production" (3.0\%), "Latin America" (3.0\%), "Impact Factor" (1.8\%), "Bibliometric Indicators" (1.8\%) and "Sciencometrics" (1.7\%). The KW with the highest degree of centrality and intermediation was "Bibliometrics". The KW with the highest degree of proximity was "Research Evaluation". Five groups of keywords behaving as cliques in the network were also found.

Keywords: Bibliometrics; Informetrics; Scientometrics; Mexico. 


\section{INTRODUCCIÓN}

—1 objetivo de este artículo es realizar un análisis de co-palabras utilizando las palabras clave en los documentos publicados sobre las metrías (bibliometría, informetría, cienciometría, patentometría, etc.) en México. El periodo escogido se extiende desde los primeros trabajos publicados en la década de 1970 hasta diciembre de 2012, un periodo extenso como para esperar que la literatura publicada se acumule, se sedimente y muestre algún tipo de organización del conocimiento bibliométrico. Una revista académica exige, para aceptar la publicación de un artículo, que el autor presente también un resumen o abstract y las palabras clave que representan adecuadamente el asunto o contenido del artículo. Estas palabras clave se han convertido en el foco de análisis de la bibliometría contemporánea.

El análisis de co-palabras estudia la co-ocurrencia de palabras en un texto, es decir, el uso de dos o más palabras conjuntas y representativas en los títulos, resúmenes, descriptores o encabezamientos de materia de un texto; en otras palabras, es el estudio de los términos que representan los conceptos contenidos en el texto de un documento en los casos en que dos o más palabras representativas aparecen juntas en el título de los artículos científicos, en el resumen o abstract, en los términos usados como palabras clave, en los descriptores o aun en el propio texto. Permite detectar clústeres, programas o líneas de investigación existentes en un determinado campo del conocimiento. Con la técnica del análisis de co-palabras es posible obtener mapas para visualizar la estructura del conocimiento de un campo científico. Los términos identificados pueden caracterizarse por conceptos de proximidad y distancia y, a su vez, representarse gráficamente, constituyendo la base para la construcción de los mapas del conocimiento de la ciencia. Estos mapas son los que representan gráficamente las proximidades y distancias de las co-palabras dando como resultado la estructura temporal de un campo del conocimiento. La distancia entre dos palabras en el mapa indica la mayor o menor relación entre ellas, pues

el fundamento metodológico del análisis de co-palabras es la idea de que la co-ocurrencia de palabras clave describe el contenido de un documento en un registro. Por lo tanto, desde el punto de vista metodológico, es una cuestión de usar uno o más índices para medir la intensidad relativa de esas ocurrencias y efectuar una representación simplificada de las redes que pueden ser evidenciadas en un gráfico. (Callon, Courtial y Laville, 1991: 160-161)

Los mapas de la ciencia son representaciones simbólicas de los campos científicos, en los cuales los elementos se distribuyen por su similitud, los más 
relacionados se sitúan más próximos y los menos relacionados se localizan más lejanos (Noyons, 2001). El análisis de la producción científica por los asuntos estudiados es un tópico en el que los mapas de la ciencia tienen mayores aplicaciones. Esa modalidad de análisis permite descubrir la evolución, la continuidad, los cambios o la extinción de líneas de investigación a lo largo del tiempo, así como indicar sus tendencias de desarrollo. En el procesamiento y análisis de los datos se utilizan software especializados y operaciones matemáticas que aplican algoritmos para la producción de listas de frecuencia de uso de las palabras y/o efectúan refinamientos de filtración retirando o eliminando palabras sin significado o superfluas. Para la visualización de los datos se utilizan técnicas bidimensionales (Noyons y Van Raan, 1998). Por lo tanto, usando la técnica de análisis de co-palabras se puede intentar establecer un mapa de las preocupaciones temáticas en la bibliometría mexicana. Se pretende dar respuesta a las siguientes cuestiones: ¿las palabras clave identifican las preocupaciones temáticas de la bibliometría mexicana? ¿Cuál es la estructura temática de la bibliometría mexicana?

Para alcanzar los objetivos propuestos, este artículo está organizado de la siguiente manera: en la primera parte se hace una introducción al problema de investigación y se formulan las preguntas de investigación. En la segunda parte se revisa la literatura pertinente. En la tercera parte se describe la metodología, es decir, las unidades de análisis, la forma de recolección de los datos y la forma de medición de los mismos. En la cuarta parte se describen los resultados obtenidos y las conclusiones. Finalmente se presenta la literatura revisada en la redacción de esta investigación.

\section{REVISIÓN DE LA LITERATURA}

Las redes pueden ser consideradas estructuras capaces de expandirse indefinidamente cuando sus actores se comunican, integrando nuevos nodos y fomentando la colaboración. Consisten de un actor que puede ser una persona, un grupo de personas, organizaciones, conceptos, enlaces u otras colectividades. Los lazos establecen las conexiones entre pares de actores (Newman, 2000; Borgatti y Foster, 2003). Entre los estudios de redes destacan el análisis de co-palabras que posibilita identificar las tendencias temáticas de un área, ya sea en el tiempo o de acuerdo con el grado de centralidad y densidad. Estos estudios también deben considerar otros aspectos relacionados con la producción del documento que incluye las palabras clave, sobre todo por lo que puede significar que un autor haya escrito un texto antes de la revolución tecnológica que se vive actualmente. Es por esto que la 
mayoría de las aplicaciones que analizan las palabras clave consideran como periodos de tiempo antes y después de 1991. Según Bourdieu (1998: 5), “[...] para comprender una obra, hay que comprender primero la producción, el campo de producción; la relación entre el campo en el cual ella se produce y el campo en el que la obra es recibida [...]”. En este caso esta afirmación es de gran importancia, sobre todo al analizar las relaciones entre nodos, así como el uso de determinadas palabras clave y desuso de otras. Es importante considerar que la tendencia de agregar a un artículo palabras clave es una práctica reciente, que se debe en gran medida a la "explosión de la información" y a la aparición de grandes bases de datos como el Web of Knowledge, Scopus, etc. También el uso de las palabras clave como forma de representación del contenido de un documento se convierte en un "código" que permite el "intercambio lingüístico" entre científicos. Este código es establecido por la misma comunidad de científicos de un área del conocimiento que ha sido "aceptado socialmente" por éstos, con base en las condiciones del "mercado" de producción simbólica. Esto significa que las palabras clave representan el "lenguaje científico" y que son usadas por un "productor autorizado" que forma parte de una élite académica que selecciona del "lenguaje autorizado" las palabras más adecuadas socialmente aceptadas para representar el contenido de un documento (Bourdieu, 2001).

La aplicación del análisis de co-palabras ha sido empleada en una diversidad de áreas del conocimiento, por ejemplo, ciencia y tecnología (Stuart y Botella, 2014); computación (Liu et al., 2014); astronomía en la Argentina (Boeris, 2012); comportamiento del consumidor (Muñoz-Leiva et al., 2011); revistas de criminología (Hogenraad, Kaminski y McKenzie, 1995), y seguridad biológica (Cambrosio et al., 1993). Estas son solamente algunas de cientos de aplicaciones en áreas diferentes a la bibliotecología y ciencia de la información.

El análisis de co-palabras en la bibliotecología y ciencia de la información se realizó por $\mathrm{Hu}$ et al. (2013), quienes estudiaron las palabras clave incluidas en 18 revistas indizadas en la base de datos Chinese Journal Fu11-Text Database (CJFTD) en el periodo de 2008 a 2012. Encontraron 24713 artículos con 80431 palabras clave, una media de 3.72 palabras clave por artículo. Hallaron que las palabras clave con un alto grado de centralidad fueron servicios de información, buscadores, servicio de referencia e investigación, entre otras. Las palabras clave con alta centralidad de intermediación fueron arquitectura de la información, gestión de la información, comportamiento de la información y gestión del conocimiento. También identificaron 63 palabras clave en el centro-periferia que representan el foco de investigación en la bibliotecología y ciencia de la información china, entre las cuales 
están servicio de información, gestión del conocimiento, intercambio de información, recursos de información, alfabetización informacional, gestión de bibliotecas, etc. Zong et al. (2013) analizaron las palabras clave de las tesis de doctorado en bibliotecología y ciencia de la información en China en el periodo de 1994 a 2011. Recolectaron los datos por medio de seis bases de datos de tesis que son de acceso público y que forman parte de los recursos de información de las universidades chinas que están autorizadas por el gobierno para otorgar grados de doctor en esta área del conocimiento. Encontraron que la Universidad de Wuhan es la institución más importante en bibliotecología y ciencia de la información que otorga títulos de doctorado en esta disciplina. Utilizaron el análisis de co-palabras y encontraron que los focos de investigación fueron recursos de información, ontología, web semántica, búsqueda semántica, gobierno electrónico, gestión de recursos de información, gestión del conocimiento, conocimiento, etc.

También Hou, Hsu y Yang (2010) analizaron el número de citas, país de publicación, temas, autores, etc. de los documentos sobre minería de textos indizados en el Social Sciences Citation Index (SSCI) del Web of Science en el periodo de 1991 a 2009. Encontraron 1319 trabajos que se centran en 10 temas, entre los cuales destacan inteligencia artificial, ciencias de la computación y sistemas de información. Ding, Chowdhury y Foo (2001) analizaron las palabras clave de 2012 documentos sobre recuperación de información indizados en la bases de datos SCI (Science Citation Index) y SSCI (Social Sciences Citation Index) en el periodo de 1987 a 1997. Estudiaron las palabras clave que aparecen indizadas en las bases de datos SCI y SSCI y también extrajeron las palabras clave más importantes de los títulos y resúmenes de forma manual. Encontraron que el número medio de palabras clave por artículo fue de 5.09 y el rango de palabras clave para cada artículo varía de uno a diez. Alrededor de $5.4 \%$ de artículos tienen 10 palabras clave, mientras que $93.4 \%$ de los artículos tienen más de una palabra clave. El periodo estudiado fue dividido en dos partes: el primero de 1987-1991 y el segundo de 19921997 , en los cuales solamente se estudiaron en total 240 palabras clave con más de dos ocurrencias en los periodos. Las palabras clave del periodo 1987 a 1991 se conjuntaron en cinco grupos; por ejemplo, el primer grupo incluye los temas de investigación relacionados con la búsqueda, análisis de sistemas, almacenamiento computarizado y recuperación, etc. El segundo grupo incluye almacenamiento y recuperación de información, medicina, catálogos en línea, etc. El tercer grupo incluye redes neuronales, almacenamiento y recuperación de imágenes, etc. El cuarto grupo incluye multimedia, Internet, aplicaciones informáticas, etc. El quinto grupo incluye almacenamiento de datos, almacenamiento de datos óptico, etc. Las palabras clave del segundo periodo 
que encontraron en el primer grupo incluyen almacenamiento y recuperación de información, búsqueda, análisis de sistemas, etc. El segundo grupo incluye redes, multimedia, medicina, Internet, etc. El tercer grupo incluye almacenamiento de datos, almacenamiento de datos ópticos, etc. El cuarto grupo incluye redes neuronales, almacenamiento y recuperación de imágenes, estilo de aprendizaje, memoria, codificación, etc. El quinto grupo incluye bibliotecas, CD-ROM, materiales de la biblioteca, bases de datos relacionales, etc.

En Croacia, Bosanac, Matešić y Toléić (2009) estudiaron los cambios temáticos en las ciencias de la información con el tiempo, con el fin de mostrar el desarrollo de este campo del conocimiento por medio del análisis de co-palabras de 367 documentos escritos por 35 autores, estos artículos contenían 689 palabras clave. Estudiaron los periodos de 1995-2002 y 2002-2009. Encontraron que durante 1995 a 2009 hubo cinco grupos principales. El primero es la museología, que incluye temas relacionados con los usuarios, los museos y establecimientos; el segundo grupo es el idioma croata relacionado con conceptos y temas de procesamiento de lenguaje natural y el campo de la inteligencia artificial; el tercer grupo es la comunicación científica que incluye temas sobre las actividades científicas; el cuarto grupo es la alfabetización informacional que contiene el conocimiento y la biblioteca, que son conceptos orientados a la administración pública, y el quinto grupo la educación, que incluye conceptos relacionados con la sociedad en red. En el primer periodo (1995-2002) hallaron 35 palabras clave que tuvieron frecuencias desde dos usos hasta 187 ocurrencias, estas palabras están representadas en tres nodos: educación, tecnología de información y comunicación de la biblioteca. En el segundo periodo (2002-2009) encontraron un total de 37 palabras clave con una frecuencia de uso de dos a 189 ocurrencias, estas palabras están representadas en cinco nodos: educación, repositorio, alfabetización en información, educación superior, información y tecnología de la comunicación.

Van den Besselaar y Heimeriks (2007) analizaron las palabras clave en el área de las ciencias de la información entre 1986 y 2002. Para realizar este análisis se basaron en los trabajos publicados en las revistas Journal of the American Society of Information Science, Journal of Documentation, Information Processing and Management, Annual Review of Information Science and Technology, Proceedings Asist, Canadian Journal of Information Science, Journal of Information Science y Scientometrics. Analizaron las palabras clave contenidas en los artículos que se publicaron en 1986, año en el que resaltan las temáticas búsqueda de información, catálogos e índices, recuperación de información, etc. En 1992 los temas centrales fueron métodos de recuperación de la información, compresión de datos, recuperación de información e hipertexto. En 1996 los temas centrales fueron búsqueda de información, 
métodos en recuperación de información, desempeño y evaluación de la recuperación, sistemas de información y algoritmos de recuperación. En 2000 los temas centrales fueron algoritmos de recuperación, búsqueda de información y búsqueda en línea. En 2002 los temas centrales fueron búsqueda de información, arquitectura de la información y recuperación de documentos. En resumen, en el periodo de 1986 a 2002 encontraron 1030 nodos en un total de 1551 artículos y reseñas que mostraron que la ciencia de la información se ha desarrollado en los subcampos de recuperación de información, cienciometría y estudios de redes. Estos autores afirman que esta área del conocimiento con el tiempo ha evolucionado en cuanto a las temáticas que se investigan.

En América Latina se encontraron distintos estudios del análisis de co-palabras en diferentes campos, como el trabajo de Silveira (2007), que analizó las palabras clave de las ponencias presentadas en la sección GT 4 de la sexta reunión ENANCIB que se lleva a cabo en Brasil. Seleccionó solamente los trabajos que incluyeran en sus temáticas gestión de información, gestión del conocimiento, información para la toma de decisiones e inteligencia competitiva. Encontró que la palabra clave gestión de la información representa el núcleo del grupo temático de esta reunión, mientras que gestión del conocimiento, información para la toma de decisiones y la inteligencia competitiva forman un conjunto de temas que se relacionan y ligan con la palabra clave núcleo, es decir, gestión de la información. Concluyó que la temática gestión del conocimiento está fuertemente ligada a la gestión de información. Liberatore y Chaves Guimarães (2012) analizaron los artículos publicados sobre gestión de la información y gestión del conocimiento, publicados en el periodo de 2000 a 2009 en las revistas Ciência da Informação, DataGramaZero, Perspectivas em Ciência da Informação y Transinformação. Hallaron un total de 98 artículos sobre estas temáticas y estudiaron la co-ocurrencia de palabras. Encontraron que ambos términos están relacionados y presentan una escasa diferencia en el campo semántico. También hallaron que la gestión del conocimiento es uno de los enfoques más usados porque es el nodo con mayor intermediación en la red de co-palabras. Las temáticas que tienen relación con la gestión de la información y la gestión del conocimiento en un primer nivel son inteligência competitiva, inovação, tecnologias da informação y profissionais da informação. En un segundo nivel están los conceptos de inclusão digital, sociedade do conhecimento, cidadania y los enfoques disciplinares desde la ciência da informação. Los temas relacionados con la gestión del conocimiento son comunicação científica, fontes de informação, bibliotecas universitárias y digitais y sistemas de informação. 
Los temas más cercanos a la organización del conocimiento son ontologias, metadados y arquivos abertos, así como la bibliometria e informetria son temas que se asocian desde una perspectiva metodológica en los distintos estudios empíricos realizados en esta área. Los temas relacionados con la gestión de la información se centran en disseminação da informação, competência informacional y los estudios de la información en general.

También se encontraron trabajos en México como el de Miranda, Licea de Arenas y Gómez-Hernández (2013), quienes estudiaron la evolución de la bibliotecología y ciencia de la información en México por medio de 106 títulos de revistas en el periodo de 1956 a 2006. Analizaron la productividad de autores, la red de co-autorías y la red de co-descriptores. La red de co-descriptores solamente la aplicaron a los trabajos publicados por Judith Licea de Arenas, quien fue la autora más productiva, y en esa red encontraron que las palabras con conexiones frecuentes fueron investigación, información y ciencias de la salud, así como los temas México, educación, colegios y universidades, bibliotecología y planes de estudio. También aplicaron la red de co-descriptores a tres revistas, entre ellas el Boletín de la Biblioteca Nacional, en el periodo de 1956-1964, y encontraron que los descriptores más frecuentes fueron México, libros, libreros, historia, bibliografía, biografías, etc. En el Anuario de Biblioteconomía y Archivonomía en el periodo 1961-1964 hallaron que los descriptores con mayor frecuencia fueron historia, educación, bibliotecología y servicios bibliotecarios, entre otros. En la revista Investigación Bibliotecológica en el periodo de 1986 a 2006 encontraron que los nodos principales fueron bibliotecología, métodos de investigación, modelos matemáticos, investigación y desarrollo, evaluación, clasificación, educación, etc. En esta red destacan por su mayor frecuencia los nodos bibliotecas públicas, información bibliotecológica, bibliotecología, investigación y ciencias, entre otros descriptores.

Como se puede ver por la literatura revisada y hasta donde es del conocimiento de los autores de este artículo, las exploraciones de este tipo de análisis de redes de co-palabras no han tomado como campo de investigación y exploración los documentos producidos en el área de la bibliometría mexicana.

\section{MATERIAL Y MÉTODOS}

Para identificar las palabras clave usadas en esos documentos se hizo una búsqueda en los títulos, palabras clave y resúmenes con múltiples combinaciones booleanas usando los términos listados en el $A$ nexo $A$ en las bases de datos bibliográficas que aparecen mencionadas en el Anexo B. Las referencias encontradas fueron 
exportadas a una base de datos bibliográfica en el software de administración de bibliografías EndNote X5. Cada uno de los trabajos encontrados se leyó, con el fin de verificar si el tema tratado tenía relación con las palabras clave usadas en esos documentos. De esas publicaciones también se revisaron las referencias bibliográficas para detectar otros trabajos sobre bibliometría, cienciometría o informetría, etc. no recuperados por medio de las bases de datos que se utilizaron. Para identificar a los autores mexicanos se hizo un seguimiento de la afiliación institucional mencionada en cada documento recuperado. Muchas veces fue necesario buscar currículos académicos en Internet para identificar y asegurarse de que eran mexicanos o extranjeros afiliados a instituciones mexicanas, así como autores mexicanos que publicaron en el extranjero y autores extranjeros que publicaron en México. En algunas ocasiones fue necesario contactarlos por medio del correo electrónico. De esa manera fueron recopilados artículos publicados en revistas académicas, cartas al editor, artículos publicados en periódicos, ponencias presentadas en eventos nacionales y/o extranjeros y capítulos de libros que trataran sobre la aplicación de las técnicas relacionadas con la bibliometría, la cienciometría, la informetría, etc. producidos por autores mexicanos y extranjeros en el país y por mexicanos en el extranjero. Se excluyeron libros, tesis y literatura gris porque estos tipos de documentos no son indizados en las bases de datos bibliográficas consultadas. Este trabajo cubre el periodo que va desde los primeros trabajos publicados en la década de 1970 hasta diciembre del 2012.

Para identificar los principales subcampos de la bibliometría mexicana se emplearon tres técnicas: el análisis de co-ocurrencia de palabras, la agrupación jerárquica y el mapa de la red social. El análisis de co-ocurrencia de palabras clave es una técnica ampliamente utilizada en bibliometría (He, 1999). La idea básica de este método es que, utilizando el análisis de conglomerados o el análisis factorial, se identifican grupos de palabras clave de alta frecuencia de co-ocurrencia en la literatura en un determinado campo científico. Después se utiliza la técnica de análisis de redes sociales y se traza un mapa de las relaciones entre las palabras clave. Por medio de los lazos de las posiciones de las palabras clave en el mapa, pueden ser fácilmente identificados los principales asuntos de investigación (Ding, Chowdhury y Foo, 2001). Para llevar a cabo el análisis de co-palabras, cuatro pasos secuenciales son generalmente implementados por los investigadores: la selección y normalización de las palabras clave, la construcción de la matriz de co-ocurrencias, el análisis de clústeres y el trazado del mapa.

Una vez obtenidas las matrices de adyacencia, se hicieron los análisis de densidad, centralidad (grado, cercanía, e intermediación) con UCINET 6. Las tablas resultantes fueron analizadas y un reporte con la interpretación de esos resultados fue creado para las co-palabras. 
Para medir la centralidad se consideraron el grado de salida (Outdeg) que tiene en cuenta las relaciones, la actividad y la capacidad de cada palabra clave para relacionarse con otras palabras clave. El grado de entrada (Indeg) indica el número de palabras clave que se relacionan de forma directa con otras y permite conocer las palabras clave más importantes dentro de la red. El grado de salida normalizado (nOutdeg) y el grado de entrada normalizado (nIndeg) son las representaciones porcentuales del inicio y recepción de las relaciones (Andrés et al., 2010; Velázquez Álvarez y Aguilar Gallegos, 2005).

\section{Resultados}

Se encontraron 459 documentos producidos y publicados hasta diciembre del 2012 que incluyen 681 palabras clave con 1914 ocurrencias, como se muestra en la Tabla 1. Las palabras clave con la mayor ocurrencia son "bibliometría" (10.61\%), "México" (6.27\%), "producción científica" (3.13\%), "América Latina" (2.66 \%), "factor de impacto" (1.83\%), "indicadores bibliométricos" $(1.78 \%$ ) y "cienciometría" (1.67 \%). 463 palabras clave ( $24.19 \%)$ tienen una única ocurrencia y 103 palabras clave (10.76\%) tienen dos ocurrencias en la literatura publicada sobre las metrías en México.

Tabla 1. Ocurrencias de las palabras clave

\begin{tabular}{|c|c|c|c|}
\hline Palabras & 0currencias & $\begin{array}{c}\text { Total de } \\
\text { ocurrencias }\end{array}$ & Porcentaje \\
\hline 1 & 203 & 203 & 10.61 \\
\hline 1 & 120 & 120 & 6.27 \\
\hline 1 & 60 & 60 & 3.13 \\
\hline 1 & 51 & 51 & 2.66 \\
\hline 1 & 35 & 35 & 1.83 \\
\hline 1 & 34 & 34 & 1.78 \\
\hline 1 & 32 & 32 & 1.67 \\
\hline 2 & 28 & 56 & 2.93 \\
\hline 1 & 25 & 25 & 1.31 \\
\hline 1 & 23 & 23 & 1.20 \\
\hline 1 & 21 & 21 & 1.10 \\
\hline 1 & 18 & 18 & 0.94 \\
\hline
\end{tabular}




\begin{tabular}{|c|c|c|c|}
\hline 3 & 17 & 51 & 2.66 \\
\hline 1 & 16 & 16 & 0.84 \\
\hline 1 & 15 & 15 & 0.78 \\
\hline 3 & 14 & 42 & 2.19 \\
\hline 2 & 11 & 22 & 1.15 \\
\hline 1 & 10 & 10 & 0.52 \\
\hline 3 & 9 & 27 & 1.41 \\
\hline 7 & 8 & 56 & 2.93 \\
\hline 6 & 7 & 42 & 2.19 \\
\hline 7 & 6 & 42 & 2.19 \\
\hline 12 & 5 & 60 & 3.13 \\
\hline 16 & 4 & 64 & 3.34 \\
\hline 40 & 3 & 120 & 6.27 \\
\hline 103 & 2 & 206 & 10.76 \\
\hline 463 & 1 & 463 & 24.19 \\
\hline 681 & & 1914 & 100 \\
\hline
\end{tabular}

La Tabla 2 muestra un comparativo de las palabras clave de mayor uso en tres periodos cronológicos, en los que "bibliometría" y "México" son las más comunes y de mayor frecuencia de uso. Estas palabras pueden ser consideradas como las marcadoras o las delimitadoras del tema y los conceptos usados en el campo de la bibliometría mexicana. Las palabras clave de menor frecuencia parecen indicar el énfasis o dirección de las investigaciones realizadas en los respectivos periodos. Por ejemplo, en el primer periodo el énfasis está en "análisis de citas" (9) y "ley de Bradford" (7). En el segundo periodo el énfasis está en "América Latina" (17), "cienciometría" (12), "factor de impacto" e "investigación científica" (10). Mientras que en el tercer periodo el énfasis aparece en "producción científica" (36), "América Latina" (31), "indicadores bibliométricos" (27), "factor de impacto" (25), etc.

Tabla 2. Número de palabras clave en el periodo de 1970 hasta 2012

\begin{tabular}{|l|c|l|l|l|c|}
\hline \multicolumn{2}{|c|}{ 1970-1990 } & \multicolumn{2}{c|}{ 1991-2000 } & \multicolumn{1}{c|}{ 2001-2012 } & \\
\hline \multicolumn{1}{|c|}{ Palabras clave } & Frecuencia & \multicolumn{1}{|c|}{ Palabras clave } & Frecuencia & Palabras clave & Frecuencia \\
\hline Bibliometría & 19 & Bibliometría & 69 & Bibliometría & 115 \\
\hline México & 11 & México & 43 & México & 66 \\
\hline Análisis de citas & 9 & América Latina & 17 & Producción científica & 36 \\
\hline
\end{tabular}




\begin{tabular}{|c|c|c|c|c|c|}
\hline Ley de Bradford & 7 & Cienciometría & 12 & América Latina & 31 \\
\hline Veterinaria & 5 & Factor de impacto & 10 & $\begin{array}{l}\text { Indicadores bibliomé- } \\
\text { tricos }\end{array}$ & 27 \\
\hline $\begin{array}{l}\text { Reproducción } \\
\text { animal }\end{array}$ & 5 & $\begin{array}{l}\text { Investigación } \\
\text { científica }\end{array}$ & 10 & Factor de impacto & 25 \\
\hline $\begin{array}{l}\text { Investigación } \\
\text { científica }\end{array}$ & 5 & Bibliotecología & 7 & Cienciometría & 17 \\
\hline UNAM & 3 & $\begin{array}{l}\text { Indicadores } \\
\text { bibliométricos }\end{array}$ & 7 & $\begin{array}{l}\text { Comunicación } \\
\text { científica }\end{array}$ & 17 \\
\hline $\begin{array}{l}\text { Productividad } \\
\text { científica }\end{array}$ & 3 & $\begin{array}{l}\text { Productividad } \\
\text { científica }\end{array}$ & 7 & Revistas académicas & 16 \\
\hline Cienciometría & 3 & $\begin{array}{l}\text { Publicaciones } \\
\text { periódicas }\end{array}$ & 7 & UNAM & 16 \\
\hline \multirow[t]{21}{*}{ América Latina } & 3 & $\begin{array}{l}\text { Ciencia de la } \\
\text { información }\end{array}$ & 6 & Bases de datos & 15 \\
\hline & & $\begin{array}{l}\text { Comunicación } \\
\text { científica }\end{array}$ & 6 & Análisis de citas & 14 \\
\hline & & Informetría & 6 & Colaboración científica & 14 \\
\hline & & UNAM & 6 & Investigación científica & 13 \\
\hline & & Análisis de citas & 5 & Revistas científicas & 13 \\
\hline & & $\begin{array}{l}\text { Producción } \\
\text { científica }\end{array}$ & 5 & Visibilidad & 13 \\
\hline & & Revistas científicas & 5 & Bibliotecología & 12 \\
\hline & & Ciencias de la salud & 4 & Análisis bibliométrico & 10 \\
\hline & & Comunicación social & 4 & El Caribe & 10 \\
\hline & & El Caribe & 4 & $\begin{array}{l}\text { Ciencia de la infor- } \\
\text { mación }\end{array}$ & 9 \\
\hline & & Ley de Bradford & 4 & Índice $h$ & 9 \\
\hline & & $\begin{array}{l}\text { Publicaciones } \\
\text { científicas }\end{array}$ & 4 & Productividad científica & 9 \\
\hline & & $\begin{array}{l}\text { Sistemas de infor- } \\
\text { mación }\end{array}$ & 4 & Física & 8 \\
\hline & & Ciencias sociales & 3 & Science Citation Index & 8 \\
\hline & & $\begin{array}{l}\text { Colaboración } \\
\text { científica }\end{array}$ & 3 & $\begin{array}{l}\text { Análisis de redes } \\
\text { sociales }\end{array}$ & 6 \\
\hline & & Cuba & 3 & Ciencia y tecnología & 6 \\
\hline & & $\begin{array}{l}\text { Modelos matemá- } \\
\text { ticos }\end{array}$ & 3 & Ciencias sociales & 6 \\
\hline & & Países en desarrollo & 3 & Informetría & 6 \\
\hline & & $\begin{array}{l}\text { Reproducción } \\
\text { animal }\end{array}$ & 3 & Política científica & 6 \\
\hline & & SIDA & 3 & Salud publica & 6 \\
\hline & & VIH & 3 & & \\
\hline
\end{tabular}


La Figura 1 muestra la red de co-palabras. Para graficar esta red solamente se tuvieron en cuenta las palabras que obtuvieron entre tres y 203 co-ocurrencias. En esta red se observa que hay cuatro nodos centrales de co-palabras que son "bibliometría", "México", "cienciometría" y "América Latina". Estas palabras clave también se repiten en los tres periodos que se analizaron anteriormente, reafirmando que actúan como marcadores y delimitadores del campo de la bibliometría mexicana. Hay varias palabras clave que son "agujeros estructurales" (Hanneman y Riddle, 2005), porque son nodos que solamente se relacionan con las palabras clave centrales. Por ejemplo, "agricultura", "evaluación de colecciones", "evaluación de revistas", etc. solamente se relacionan con la palabra clave "bibliometría".

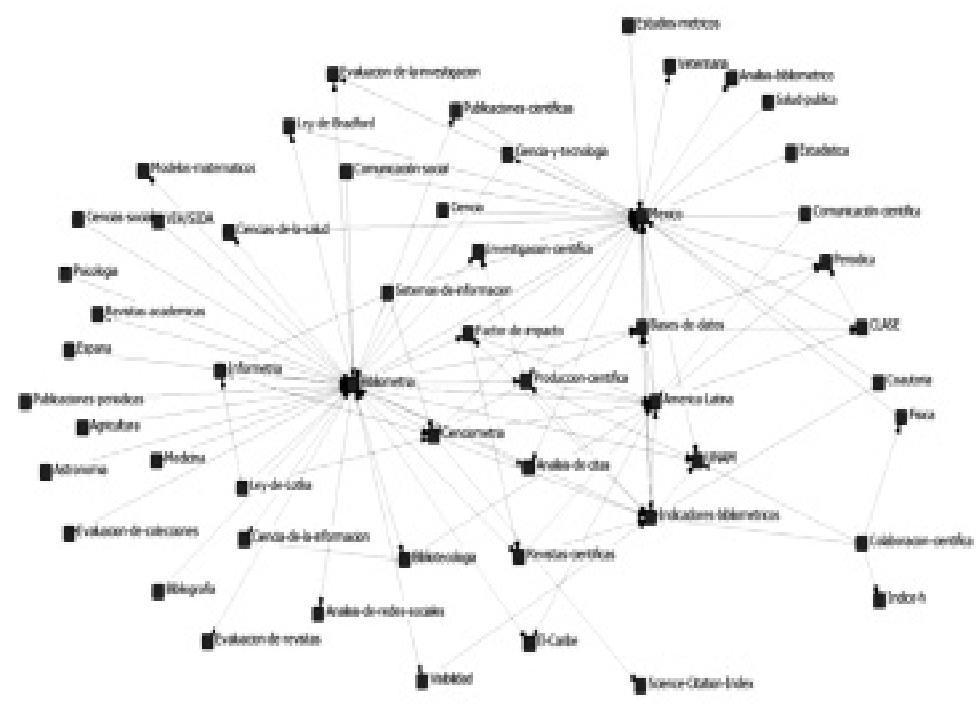

Figura 1. Red de c0-palabras

La Tabla 3 muestra el grado de centralidad de la red de co-palabras que permite identificar las palabras clave más relevantes, pero sólo se presentan aquellas palabras que alcanzaron 10.00 o más grados de salida. El grado de salida (Outdeg) de una palabra clave indica el número de conexiones que salen o parten desde una determinada palabra clave (PC), es decir, explicita la cantidad de conexiones que tiene la PC con otras palabras clave en el conjunto de documentos indizados. El grado de entrada (Indeg) de una palabra clave indica el número de conexiones que recibe una determinada palabra clave provenientes de otras PC; se refiere al número de veces que esta PC es 
procurada para ser indizada junto a otras PC en el conjunto de documentos indizados sobre el asunto bibliometría mexicana.

Por ejemplo, la palabra clave "bibliometría" tiene un grado de salida igual a 179.000 (9.059 \% de grado de salida normalizado), esto significa que esta palabra clave se revela como el nodo central de la red y actúa como fuente principal para la interacción y vinculación con otras palabras clave en los 459 documentos analizados. Tiene también un grado de entrada igual a 147.000 (7.439 \% de grado de entrada normalizado) que indica que esta PC ha sido buscada 147 veces para ser indizada junto a otras PC en el mismo conjunto de documentos analizados.

La palabra clave "México" tiene un grado de salida igual a 71.000 (3.593\% de grado de entrada normalizado) y un grado de entrada igual a 122.000 (6.174 \% de grado de entrada normalizado). El grado de salida indica también que es la segunda PC de interconexión con las otras palabras clave de la red de co-palabras, aunque como su grado de entrada es mayor que el de salida, significa que esta palabra clave es más buscada por otras palabras clave para aparecer indizadas acompañadas en el grupo de 459 documentos analizados. En general, estas palabras clave que tienen los valores más altos en el grado de centralidad son consideradas menos dependientes y tienen más vínculos en la red, lo que les permite aparecer con más frecuencia junto a otras palabras clave y son las más influyentes en esta red de co-palabras.

Tabla 3. Centralidad de las palabras clave

\begin{tabular}{|c|c|c|c|c|c|}
\hline \multirow{2}{*}{\multicolumn{2}{|c|}{ Palabras clave }} & 1 & 2 & 3 & 4 \\
\hline & & Outdeg & Indeg & nOutdeg & nlndeg \\
\hline 9 & Bibliometría & 179.000 & 147.000 & 9.059 & 7.439 \\
\hline 38 & México & 71.000 & 122.000 & 3.593 & 6.174 \\
\hline & Producción-científica & 49.000 & 25.000 & 2.480 & 1.265 \\
\hline 2 & América-Latina & 38.000 & 31.000 & 1.923 & 1.569 \\
\hline & Cienciometría & 22.000 & 32.000 & 1.113 & 1.619 \\
\hline 31 & Indicadores-bibliométricos & 22.000 & 31.000 & 1.113 & 1.569 \\
\hline & Bibliotecología & 17.000 & 5.000 & 0.860 & 0.253 \\
\hline 29 & Factor-de-impacto & 17.000 & 7.000 & 0.860 & 0.354 \\
\hline 19 & Colaboración-científica & 16.000 & 0.000 & 0.810 & 0.000 \\
\hline 49 & Sistemas-de-información & 13.000 & 0.000 & 0.658 & 0.000 \\
\hline
\end{tabular}




\begin{tabular}{|c|c|c|c|c|c|}
\hline & Ley-de-Lotka & 12.000 & 0.000 & 0.607 & 0.000 \\
\hline 7 & Bases-de-datos & 10.000 & 14.000 & 0.506 & 0.709 \\
\hline 15 & Ciencias-de-la-salud & 10.000 & 3.000 & 0.506 & 0.152 \\
\hline 21 & Comunicación-social & 10.000 & 0.000 & 0.506 & 0.000 \\
\hline 46 & Revistas-científicas & 10.000 & 6.000 & 0.506 & 0.304 \\
\hline 50 & UNAM & 10.000 & 19.000 & 0.506 & 0.962 \\
\hline
\end{tabular}

La Tabla 4 presenta las medidas estadísticas descriptivas del grado de centralidad. La media del grado de salida fue de 11.546 y del grado de entrada fue de 11.547, lo que significa que las PC en media conectan con 11 palabras clave. El número máximo de conexiones que salen de la red de 179 PC es propiciada por la PC "bibliometría". Estos valores son confirmados por el grado de entrada normalizado que aparece con el mismo valor que el grado de centralidad de entrada (0.584). El mínimo de conexiones fue de cero, lo que indica que existen PC que no tienen conexiones y mantienen un estado de pasividad. Las palabras clave con el mayor valor grado de salida son "bibliometría", "México", "producción científica", "América Latina", "cienciometría", "indicadores bibliométricos", "bibliotecología", "factor de impacto", "colaboración científica", "sistemas de información" y "ley de Lotka". Las palabras clave con el mayor grado de entrada son "bibliometría", "México", "producción científica", "América Latina", "cienciometría" e "indicadores bibliométricos".

El coeficiente de variación (desviación estándar/media $\times 100$ ) para el grado de entrada es del $44.63 \%$ y para el grado de salida es del $43.69 \%$, lo que indica que esta red es bastante homogénea en sus conexiones y vinculaciones de PC. La suma de todas las relaciones posibles en la red es igual a 612. El índice de centralización de salida es igual al $8.3 \%$, lo que indica la existencia de muy pocas PC centrales, y el índice de centralización de salida igual a $6.7 \%$ indica que esta red no está fuertemente centralizada y que las ventajas de posición de las PC son más o menos uniformes.

Tabla 4. Medidas estadísticas del grado de centralidad

\begin{tabular}{|l|c|c|c|c|}
\hline \multirow{2}{*}{\multicolumn{1}{|c|}{ Medidas }} & $\mathbf{1}$ & $\mathbf{2}$ & $\mathbf{3}$ & $\mathbf{4}$ \\
\cline { 2 - 5 } & OutDegree & InDegree & NrmoutDeg & NrmInDeg \\
\hline Media & 11.546 & 11.547 & 0.584 & 0.584 \\
\hline Desviación estándar & 26.422 & 25.872 & 1.337 & 1.309 \\
\hline
\end{tabular}




\begin{tabular}{|l|c|c|c|c|}
\hline Varianza & 698.097 & 669.380 & 1.788 & 1.714 \\
\hline Valor mínimo & 0.000 & 0.000 & 0.000 & 0.000 \\
\hline Valor máximo & 179.000 & 147.000 & 9.059 & 7.439 \\
\hline Norma Euclidiana & 209.919 & 206.262 & 10.623 & 10.438 \\
\hline
\end{tabular}

La Tabla 5 muestra el grado de intermediación de las palabras clave que forman la red de co-palabras. La intermediación muestra el grado en que las PC actúan como puentes/intermediarios frente a otra(s) palabra(s) clave de la red, es decir, los actores centrales que se convierten en los intermediarios para facilitar el flujo de información en la red de co-palabras "son puentes que se pueden aprovechar en estrategias refuerzo, enfocadas al incremento del compromiso y cohesión de la red" (Galindres, Soto Mejía y Caro Isaza, 2013: 495), es decir, que la intermediación es "un parámetro" que permite comprender las distancias geodésicas que hay entre una palabra clave con otras palabra clave de la misma red (Galindres, Soto Mejía y Caro Isaza, 2013: 493). Analizando todas las PC se identifica a la PC que se localiza en el camino más corto entre otras PC. Cuantas más PC se colocan en la ruta más corta frente a otras PC mayor será su intermediación y esa PC será vista más como una PC "intermediaria”. Altos valores de intermediación indican que otras PC tienen que "pasar" por medio de esta PC para conectar a otras PC. Las PC que se colocan en los caminos más cortos entre pares de PC son vistas como las más centrales en la red de co-palabras, pues más PC dependen de ellas para realizar conexiones con el resto de las PC de la red.

Se identificaron solamente 11 palabras clave que son intermediarias o que facilitan la comunicación en esta red. La palabra clave con el mayor grado de intermediación es "bibliometría", con capacidad de intermediar otras 962.474 palabras clave. Esta palabra clave es la más importante, ya que es la que controla la comunicación, mantiene la cohesión y las distancias entre las otras 962.474 palabras clave. También se encuentra la palabra clave "México", con capacidad de intermediar otras 485.033 PC y que en orden de importancia en esta red de co-palabras ocupa el segundo lugar. Otras palabras clave que tienen valores por encima del promedio y que también mantienen la comunicación y la cohesión de la red son "indicadores bibliométricos” con 86.140, “América Latina” con 68.548, “cienciometría” con 56.679. Mientras que de 53 nodos que conforman la red de co-palabras, 42 palabras clave no tienen comunicación o intermediación con otras PC. 
Tabla 5. Grado de intermediación

\begin{tabular}{|l|c|c|}
\hline \multirow{2}{*}{\multicolumn{1}{|c|}{ Palabras clave }} & $\mathbf{1}$ & $\mathbf{2}$ \\
\cline { 2 - 3 } & Betweenness & nBetweenness \\
\hline 9 Bibliometría & 962.474 & 36.292 \\
\hline 38 México & 485.033 & 18.289 \\
\hline 31 Indicadores-bibliométricos & 86.140 & 3.248 \\
\hline 2 América-Latina & 68.548 & 2.585 \\
\hline 17 Cienciometría & 56.679 & 2.137 \\
\hline 7 Bases-de-datos & 31.619 & 1.192 \\
\hline 41 Producción-científica & 15.874 & 0.599 \\
\hline 50 UNAM & 14.200 & 0.535 \\
\hline 30 Física & 3.100 & 0.117 \\
\hline 29 Factor-de-impacto & 3.000 & 0.113 \\
\hline 46 Revistas-científicas & 0.333 & 0.013 \\
\hline
\end{tabular}

La PC "bibliometría" (36.292 \%) con el mayor grado de intermediación está al doble de distancia de la PC "México" (18.289 \%) y ésta a su vez está seis veces más alejada que la tercera PC "indicadores bibliométricos" (3.248 \%). El resto de las PC tienen poca capacidad de intermediar o reconectar a otras PC.

La Tabla 6 muestra las medidas estadísticas descriptivas del grado de intermediación. La media de intermediación fue de 32.585 PC, es decir, se intermedian un promedio de 32.585 PC en la red. Los valores mínimos y máximos varían de cero a 962.474 con un coeficiente de variación del $22.4 \%$. Esto indica que las palabras clave que controlan la comunicación son bastante heterogéneas y dispersas.

Tabla 6. Medidas estadísticas del grado de intermediación

\begin{tabular}{|l|c|c|}
\hline \multirow{2}{*}{\multicolumn{1}{|c|}{ Medidas }} & $\mathbf{1}$ & $\mathbf{2}$ \\
\cline { 2 - 3 } & Betweenness & nBetweenness \\
\hline Promedio & 32.585 & 1.229 \\
\hline Desviación estándar & 145.508 & 5.487 \\
\hline Varianza & 21172.502 & 30.104 \\
\hline Valor mínimo & 0.000 & 0.000 \\
\hline
\end{tabular}




\begin{tabular}{|l|c|c|}
\hline Valor máximo & 962.474 & 36.292 \\
\hline Norma Euclidiana & 1085.549 & 40.933 \\
\hline
\end{tabular}

La Tabla 7 muestra las palabras clave con el mayor grado de cercanía en esta red de co-palabras. La cercanía se define como la capacidad que tiene una palabra clave para llegar a las demás palabras clave (Velázquez Álvarez y Aguilar Gallegos, 2005). Estima la distancia existente entre una PC hasta las otras PC de la red. Los mayores valores sugieren una mayor capacidad de colaboración entre PC y los valores más bajos sugieren que aquellas PC van a tener dificultades para conectarse con otras PC. Para medir la cercanía se consideran los valores de cercanía de entrada (inCloseness), cercanía de salida (outCloseness), lejanía de entrada (inFarness) y lejanía de salida (outFarness). Por ejemplo, la palabra clave que tiene el mayor valor de cercanía es "evaluación de la investigación", cuya inCloseness es igual a 7.82; esto significa que esta palabra clave es la que tiene la menor distancia para interactuar con otras palabras clave. Sin embargo, también tiene un bajo outCloseness (1.887), indicando que no puede ser rápidamente conectable (alcanzable) por las otras PC de la red. Otras palabras clave que tienen un valor alto de cercanía son "evaluación de revistas", "publicaciones periódicas", "España", "análisis de redes sociales", "modelos matemáticos", "evaluación de colecciones" y "medicina", que tienen una cercanía de entrada igual a 7.67, pero de acuerdo a los bajos valores del outCloseness (1.887) tampoco podrán ser rápidamente alcanzables por las otras PC de la red. Caso contrario sucede con las PC "bibliometría", "México", "indicadores bibliométricos", "UNAM", "cienciometría", que a pesar de tener un menor valor de inCloseness $( \pm 7.6$ a $7.3)$ tienen un mayor valor de outCloseness ( \pm 5.2 a 5.1 .

Tabla 7. Cercanía

\begin{tabular}{|l|c|c|c|c|}
\hline \multicolumn{1}{|c|}{ Palabras clave } & Farness & outFarness & inCloseness & outCloseness \\
\hline Evaluación de la investigación & 665 & 2756 & 7.82 & 1.887 \\
\hline Evaluación de revistas & 678 & 2756 & 7.67 & 1.887 \\
\hline Publicaciones-periódicas & 678 & 2756 & 7.67 & 1.887 \\
\hline España & 678 & 2756 & 7.67 & 1.887 \\
\hline Análisis de redes sociales & 678 & 2756 & 7.67 & 1.887 \\
\hline Modelos matemáticos & 678 & 2756 & 7.67 & 1.887 \\
\hline Evaluación de colecciones & 678 & 2756 & 7.67 & 1.887 \\
\hline Medicina & 678 & 2756 & 7.67 & 1.887 \\
\hline
\end{tabular}




\begin{tabular}{|c|c|c|c|c|}
\hline Análisis bibliométricos & 683 & 2756 & 7.613 & 1.887 \\
\hline Veterinaria & 683 & 2756 & 7.613 & 1.887 \\
\hline Bibliometría & 690 & 994 & 7.536 & 5.231 \\
\hline México & 695 & 1013 & 7.613 & 5.133 \\
\hline Science-Citation-Index & 700 & 2756 & 7.482 & 1.887 \\
\hline Indicadores bibliométricos & 708 & 1020 & 7.345 & 5.098 \\
\hline UNAM & 711 & 1023 & 7.314 & 5.083 \\
\hline Cienciometría & 712 & 1020 & 7.303 & 5.098 \\
\hline Producción científica & 713 & 1014 & 7.293 & 5.128 \\
\hline Investigación científica & 715 & 1046 & 7.273 & 4.971 \\
\hline Análisis de citas & 716 & 1023 & 7.263 & 5.083 \\
\hline América Latina & 718 & 1017 & 7.242 & 5.113 \\
\hline Bases de datos & 721 & 1039 & 7.212 & 5.005 \\
\hline Factor de impacto & 725 & 1019 & 7.172 & 5.103 \\
\hline Informetría & 726 & 1025 & 7.163 & 5.073 \\
\hline El Caribe & 727 & 1027 & 7.153 & 5.063 \\
\hline Publicaciones científicas & 728 & 1045 & 7.143 & 4.976 \\
\hline Visibilidad & 728 & 1052 & 7.143 & 4.943 \\
\hline Revistas científicas & 728 & 1023 & 7.143 & 5.083 \\
\hline Ciencia de la información & 728 & 1017 & 7.143 & 5.063 \\
\hline Ley de Bradford & 728 & 1045 & 7.143 & 4.976 \\
\hline Bibliotecología & 729 & 1023 & 7.133 & 5.083 \\
\hline Ciencias de la salud & 729 & 1023 & 7.133 & 5.083 \\
\hline Revistas académicas & 729 & 1027 & 7.133 & 5.063 \\
\hline Ciencia y tecnología & 733 & 1026 & 7.094 & 5.068 \\
\hline Periódica & 749 & 1044 & 6.943 & 4.981 \\
\hline CLASE & 752 & 1041 & 6.915 & 4.995 \\
\hline Índice $h$ & 2704 & 2756 & 1.923 & 1.887 \\
\hline Física & 2704 & 995 & 1.923 & 5.226 \\
\hline Astronomía & 2756 & 972 & 1.887 & 5.328 \\
\hline Ciencia & 2756 & 972 & 1.887 & 5.350 \\
\hline Comunicación social & 2756 & 972 & 1.887 & 5.350 \\
\hline Ciencias sociales & 2756 & 976 & 1.887 & 5.328 \\
\hline Ley de Lotka & 2756 & 973 & 1.887 & 5.344 \\
\hline Estudios métricos & 2756 & 995 & 1.887 & 5.226 \\
\hline Coautoría & 2756 & 989 & 1.887 & 5.258 \\
\hline
\end{tabular}




\begin{tabular}{|l|l|l|l|l|}
\hline Colaboración científica & 2756 & 893 & 1.887 & 5.823 \\
\hline Bibliografía & 2756 & 976 & 1.887 & 5.328 \\
\hline Salud pública & 2756 & 995 & 1.887 & 5.226 \\
\hline Psicología & 2756 & 976 & 1.887 & 5.328 \\
\hline Sistemas de información & 2756 & 969 & 1.887 & 5.366 \\
\hline Estadística & 2756 & 995 & 1.887 & 5.226 \\
\hline VIH/SIDA & 2756 & 976 & 1.887 & 5.238 \\
\hline Comunicación científica & 2756 & 987 & 1.887 & 5.268 \\
\hline Agricultura & 2756 & 976 & 1.887 & 5.328 \\
\hline
\end{tabular}

La Tabla 8 muestra las medidas estadísticas descriptivas del grado de cercanía. La distancia promedio de las PC para inCloseness es 5.491 y para el outCloseness es de 4.430, significa que en promedio las PC pueden ser rápidamente alcanzables por otras PC, pero éstas no pueden alcanzar rápidamente a las PC relevantes de la red. La desviación estándar y la media indican si la red es homogénea o heterogénea en relación a su capacidad de variabilidad. El coeficiente de variación de cercanía de entrada (inFarness) es del 68.95 y el de cercanía de salida (outFarness) es de 52.26; estos valores indican que hay heterogeneidad en la población de palabras clave de los documentos analizados.

Tabla 8. Medidas de estadística descriptiva del grado de cercanía

\begin{tabular}{|l|c|c|c|c|}
\hline \multicolumn{1}{|c|}{ Medidas } & inFarness & outFarness & inCloseness & outCloseness \\
\hline Promedio & 1402.245 & 1402.245 & 5.491 & 4.430 \\
\hline Desviación estándar & 966.916 & 732.887 & 2.589 & 1.383 \\
\hline Varianza & 934925.813 & 537122.813 & 6.704 & 1.914 \\
\hline Valor mínimo & 665 & 893 & 1.887 & 1.887 \\
\hline Valor máximo & 1400.245 & 1400.245 & 5.491 & 4.430 \\
\hline Norma Euclidiana & 12400.183 & 1158.723 & 44.195 & 33.784 \\
\hline
\end{tabular}

Un clique es un subconjunto de palabras clave "que están más fuertemente conectadas mutuamente que [otras palabras clave que forman parte de la red de co-palabras]" (Hanneman, 2005: 117). El clique o subgrafo es un conjunto de nodos o palabras clave que tienen vínculos entre ellos. Para que se forme un clique de palabras clave tiene que haber más de dos palabras clave. Los grupos formados por las palabras clave que representan todas las relaciones 
posibles se denominan "subgrafo máximo completo" (Quiroga, 2003: 44). En general, el análisis de los cliques de la red de co-palabras permite identificar las relaciones de una palabra clave con otras palabras clave o los grupos que forman esas palabras clave (Andrés et al., 2010; Quiroga, 2003). Los cliques son pequeños componentes cohesionados que están dentro de la red, en este caso subgrupos de palabras clave (Hanneman, 2005). La Figura 2 muestra cinco cliques o grupos identificados como centro neurálgico de la red, es decir, que cohesionan la estructura de la bibliometría mexicana; son las palabras clave que están más cerca y unidas entre sí, y forman subgrupos o asociaciones entre ellas.

1. Análisis-de-citas / Bibliometría / México

2. Bibliometría / México / Producción-científica

3. Bibliometría / México / UNAM

4. Bibliometría / Cienciometría / Informetría

5. Bibliometría / Indicadores-bibliométricos / Producción-científica

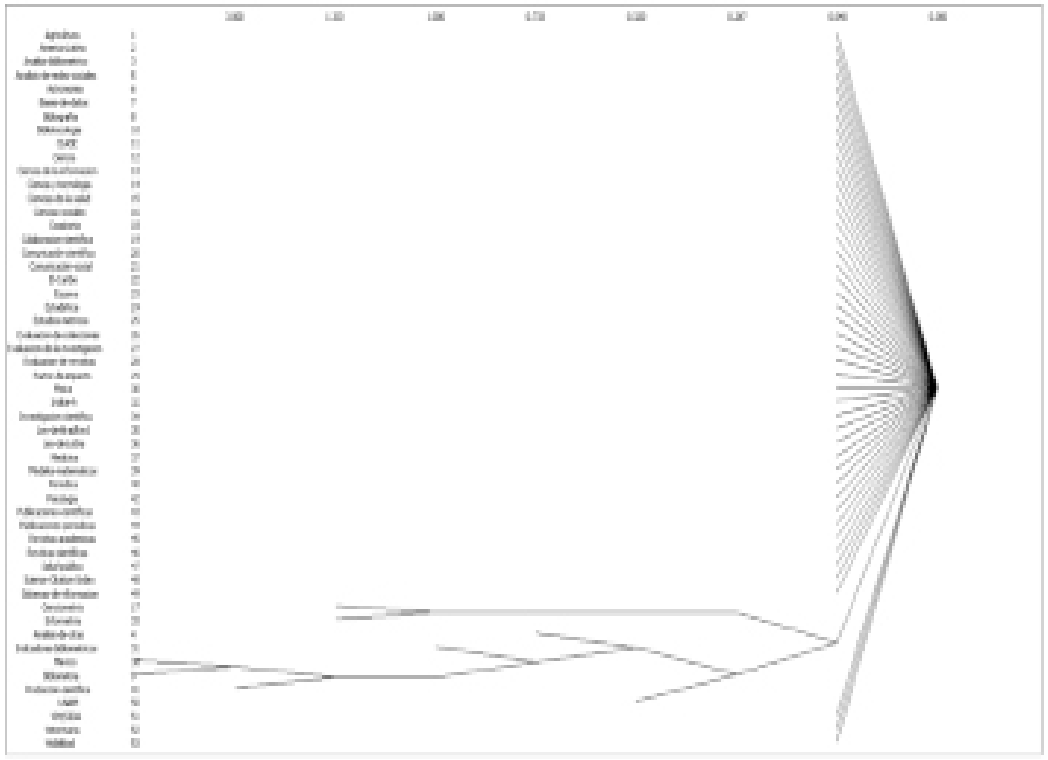

Figura 2. Cliques en la red de co-palabras 


\section{Conclusiones}

Se encontraron 459 documentos producidos y publicados hasta diciembre del 2012. Estos documentos usaron 681 palabras clave con 1914 ocurrencias. Las palabras clave identificadas con la mayor ocurrencia son "bibliometría" (10.61 \%), "México" (6.27\%), "producción científica" (3.13\%), "América Latina" (2.66 \%), "factor de impacto" (1.83\%), "indicadores bibliométricos" $(1.78 \%)$ y "cienciometría" (1.67 \%). Por otro lado, 103 palabras clave $(10.76 \%)$ tienen dos ocurrencias y 463 palabras clave $(24.19 \%)$ tienen una única ocurrencia.

Se hizo un análisis en tres periodos cronológicos y las palabras clave "bibliometría” y "México" son las más comunes y de mayor frecuencia de uso. Estas palabras pueden ser consideradas como las marcadoras o las delimitadoras del tema y los conceptos usados en el campo de la bibliometría mexicana. Las palabras clave de menor frecuencia parecen indicar el énfasis o dirección de las investigaciones realizadas en los respectivos periodos. Por ejemplo, en el primer periodo el énfasis está en "análisis de citas" (9) y "ley de Bradford" (7), en el segundo en un acercamiento hacia "América Latina" (17), "cienciometría" (12), "factor de impacto" e "investigación científica" (10). En el tercer periodo el énfasis aparece en "producción científica” (36), nuevamente "América Latina" (31), “indicadores bibliométricos” (27) y "factor de impacto” (25).

En relación al grado de centralidad se encontró que la PC "bibliometría" ha buscado 179 veces otras PC para indizar un documento revelándose como el nodo central de la red y que actúa como fuente principal para la interacción y vinculación con otras palabras clave en los 459 documentos analizados. También ha sido buscada 147 veces para ser indizada junto a otras PC en el mismo conjunto de documentos analizados. Junto a esta PC aparecen otras como "México", que ha buscado 71 veces otras PC y ha sido contactada 122 veces por otras PC. Estas dos palabras clave tienen los valores más altos en el grado de centralidad, por lo que son consideradas menos dependientes, tienen más vínculos en la red lo que les permite aparecer con más frecuencia junto a otras palabras clave y son las más influyentes en la red de co-palabras de la BM. Otras PC importantes en grados de salida son "producción científica”, "América Latina”, "cienciometría”, “indicadores bibliométricos”, "bibliotecología", "factor de impacto", "colaboración científica", "sistemas de información" y "ley de Lotka”. Las palabras clave con el mayor grado de entrada son "bibliometría”, "México”, "producción científica”, "América Latina", "cienciometría" e "indicadores bibliométricos". 
En relación con el grado de intermediación se identificaron 11 palabras clave intermediarias que facilitan la comunicación en esta red. La palabra clave con el mayor grado de intermediación es "bibliometría", con capacidad de intermediar otras 963 palabras clave. La palabra clave "México" tiene capacidad de intermediar $485 \mathrm{PC}$, que en orden de importancia en esta red de co-palabras ocupa el segundo lugar. Otras palabras clave importantes para la manutención de la comunicación y la cohesión de la red son "indicadores bibliométricos", "América Latina" y "cienciometría". Asimismo, de 53 nodos que conforman la red de co-palabras, 42 palabras clave no tienen comunicación o intermediación con otras PC.

En relación con el grado de cercanía se encontró que la palabra clave que tiene el mayor valor de cercanía es "evaluación de la investigación", lo que significa que tiene la menor distancia para interactuar con otras palabras clave. Sin embargo, también tiene un bajo outCloseness, lo que indica que no puede ser rápidamente conectable por las otras PC de la red. Otras palabras clave que tienen un valor alto de cercanía son "evaluación de revistas", "publicaciones periódicas", "España”, "análisis de redes sociales", "modelos matemáticos", "evaluación de colecciones" y "medicina", que tienen una cercanía de entrada igual a 7.67, pero de acuerdo a los bajos valores del outCloseness tampoco podrán ser rápidamente alcanzables por las otras PC de la red. Caso contrario sucede con las PC "bibliometría", "México", "indicadores cienciométricos", "UNAM" y "cienciometría", que a pesar de tener un menor valor de inCloseness tienen un mayor valor de outCloseness. También se encontraron cinco grupos de palabras clave que se comportan como cliques en la red.

\section{REFERENCIAS}

Andrés, Carlos, Daniel Getino, Héctor Ortega y Anuar Salim. 2010. "Estudio de datos de redes sociales (SNA)". Técnicas y herramientas (THAI), Mui-Tic, Universidad de Valladolid, 5 de octubre de 2010. Fecha de consulta: 2 de diciembre de 2014. http://www.gsic.uva.es/wikis/amartine/images/f/fa/SNA_informeGrupo4.pdf

Boeris, Claudia E. 2012. "Aplicación de técnicas de análisis de redes sociales y de coocurrencia de palabras en la determinación de frentes de investigación”. Artículo enviado al Boletín de la Asociación Argentina de Astronomía y presentado como póster en la 55a Reunión Nacional de la Asociación llevada a cabo en Mar de la Plata del 17 al 21 de septiembre de 2012.

Borgatti, S. P. y P. C. Foster. 2003. "The network paradigm in organizational research: a review and Typology". Journal of Management 29 (6): 991-1013.

Bosanac, Siniša, Marija Matešić y Nino Tolić . 2009. "Telling the future of information sciences: co-word analysis of keywords in scientific literature produced at 
the Department of Information Sciences in Zagreb”. INFuture2009: “Digital Resources and Knowledge Sharing”, 4-6 November 2009.

Bourdieu, Pierre. 1998. “¿Qué es hacer hablar a un autor? a propósito de Michel Foucault”. En Isabel Jiménez (comp. y trad.), Capital cultural, escuela y espacio social, 5-11. Madrid: Siglo XXI Editores.

Bourdieu, Pierre. 2001. ¿Qué significa hablar? Madrid: Akal.

Callon, M., J. P. Courtial y F. Laville. 1991. "Word analysis as a tool for describing the network of interactions between basic and technological research: the case of polymer chemistry”. Scientornetrics 22 (1): 155-205.

Cambrosio, A., C. Limoges, J. P. Courtial y F. Laville. 1993. "Historical scientometrics? Mapping over 70 years of biological safety research with coword analysis". Scientometrics 27: 119-143.

Ding, Y., G.G. Chowdhury y S. Foo. 2001. "Bibliometric cartography of information retrieval research by using co-word analysis". Information Processing \& Management 37: 817-842.

Galindres, Diego Armando, José Adalberto Soto Mejía y Carlos Arturo Caro Isaza. 2013. "Sociedad en movimiento: un análisis de redes". Scientia et Technica 18 (3) (octubre): 490-497.

Hanneman, Robert A. y M. Riddle. 2005. Introduction to social network methods. Fecha de consulta: 10 de abril de 2015. http://faculty.ucr.edu/ hanneman/nettext

Hanneman, Robert A. 2005. Introducción a los métodos del análisis de redes sociales. Universidad de California Riverside, Departamento de Sociología. Fecha de consulta: 9 de diciembre de 2014. http://revista-redes.rediris.es/webredes/text. htm

He, Qin (1999), “Knowledge discovery Through co-word analysis”. Library Trends 48 (1): 133-159.

Hogenraad, Robert, Dan Kaminski y Dean McKenzie. 1995. "Trails of social science: the visibility of scientific change in criminological journals". Social Science Information 34 (4): 663-685.

Hou, Oliver C. L., Heigen Hsu y Jiann-Min Yang. 2010. “An empirical investigation of research productivity on text mining in bibliometrics view". New Trends in Information Science and Service Science (NISS), 2010 4th International Conference on 11-13 May 2010, pp. 646-650.

$\mathrm{Hu}$, Chang-Ping, Ji-Ming Hu, Sheng-Li Deng y Yong Liu. 2013. "A co-word analysis of library and information Science in China". Scientometrics 97: 369-382.

Liberatore, Gustavo y José Augusto Chaves Guimarães. 2012. "El área de la gestión de información y del conocimiento como frente de investigación en la ciencia de la información brasileña: análisis de la producción científica en el período 20002009”. Perspectivas em Gestão \& Conhecimento, João Pessoa, 2 (2): 134-142.

Liu, Yong, Jorge Goncalves, Denzil Ferreira, Bei Xiao, Simo Hosio y Vassilis Kostakos. 2014. CHI 1994-2013: Mapping Two Decades of Intellectual Progress through Co-word Analysis. Fecha de consulta: 3 de julio de 2014. http://www. ee.oulu.fi/ vassilis/files/papers/chi14.pdf

Miranda, Israel, Judith Licea de Arenas y José Antonio Gómez-Hernández. 2013. "Publicaciones periódicas en biblioteconomía, bibliotecología, ciencias de la información y documentación en México: tendencias temáticas, productividad y 
redes de coautoría: 1956-2006”. Revista Interamericana de Bibliotecología 36 (2): 97-108.

Muñoz-Leiva, Francisco, María Isabel Viedma-del-Jesús, Juan Sánchez-Fernández y Antonio Gabriel López-Herrera. 2011. "An application of co-word analysis and bibliometric maps for detecting the most highlighting themes in the consumer behaviour research from a longitudinal perspective”. Quality \& Quantity 46 (4) (agosto): 1077-1095.

Newman, M. E. J. 2000. Who is the best connected scientist?: a study of scientific coauthorship networks. Santa Fé: The Santa Fé Institute. Paper 00-12-064.

Noyons, E. 2001. "Bibliometric mapping of science in a policy context". Scientometrics 50 (1): 83-9.

Noyons, E. C. M. y A. F. J. Van Raan. 1998. "Advanced mapping of science and technology”. Scientometrics 41 (1-2): 61-67.

Quiroga, Águeda. 2003. Introducción al análisis de datos reticulares: prácticas con UCINET6 y NetDraw1: Versión 2. Barcelona: Departamento de Ciencias Políticas, Universidad Pompeu Fabra.

Silveira, Murilo Artur Araújo da. 2007. "Gestão da informação e do conhecimento: análise temática dos trabalhos do VI ENANCIB”. Informação \& Informação 12 (2): 1-11.

Stuart, Keith, y Ana Botella. 2014. Lingüística de corpus, análisis de redes y matrices de co-ocurrencias. Fecha de consulta: 14 de junio de 2014. http://www.um.es/lacell/aelinco/contenido/pdf/41.pdf

Van den Besselaar, Peter y Gaston Heimeriks. 2007. "Mapping research topics using word-reference co-occurrences: A method and an exploratory case study". Scientometrics 68 (3): 377-393.

Velázquez Álvarez, O. Alejandro y Norman Aguilar Gallegos. 2005. Manual introductorio al análisis de redes sociales, junio de 2005. Fecha de consulta: 2 de diciembre de 2014. http://revista-redes.rediris.es/webredes/talleres/Manual_ ARS.pdf

Zong, Qian-Jin, Hong-Zhou Shen, Qin-Jian Yuan, Xiao-Wei Hu, Zhi-Ping Hou y Shun-Guo Deng. 2013. "Doctoral dissertations of library and information science in China: a co-word analysis". Scientometrics 94: 781-799.

Anexo A. Términos de busca

\begin{tabular}{|c|}
\hline México \\
\hline Índice $h$ \\
\hline Elitismo \\
\hline Frente de investigación \\
\hline Reglas 80/20 \\
\hline Obsolescencia de la literatura \\
\hline Crecimiento de la literatura \\
\hline Vida media \\
\hline
\end{tabular}




\begin{tabular}{|c|}
\hline Teoría epidémica \\
\hline Visibilidad \\
\hline Índice de Pratt \\
\hline Índice de Price \\
\hline Ley de Price \\
\hline Indicadores bibliométricos \\
\hline Ley de Goffman \\
\hline Ley de Bradford \\
\hline Ley de Lotka \\
\hline Ley de Zipf \\
\hline Punto de trasición \\
\hline Colegios invisibles \\
\hline Factor de impacto \\
\hline Factor de inmediatismo \\
\hline Análisis de citas \\
\hline Acoplamiento bibliográfico \\
\hline Co-citación \\
\hline Redes sociales \\
\hline Co-autoría \\
\hline Colabaoración científica \\
\hline Índice de colaboración \\
\hline Coeficiente de colaboración \\
\hline Circulación de la colección \\
\hline Núcleo básico de periódicos \\
\hline Indicadores en ciencia y tecnología \\
\hline Bibliometría \\
\hline Cienciometría \\
\hline Informetría \\
\hline Patentometría \\
\hline Arquivometría \\
\hline Bio-bibliometría \\
\hline Librometría \\
\hline Tecnometría \\
\hline Webometría \\
\hline
\end{tabular}


Anexo B. Bases de datos consultadas

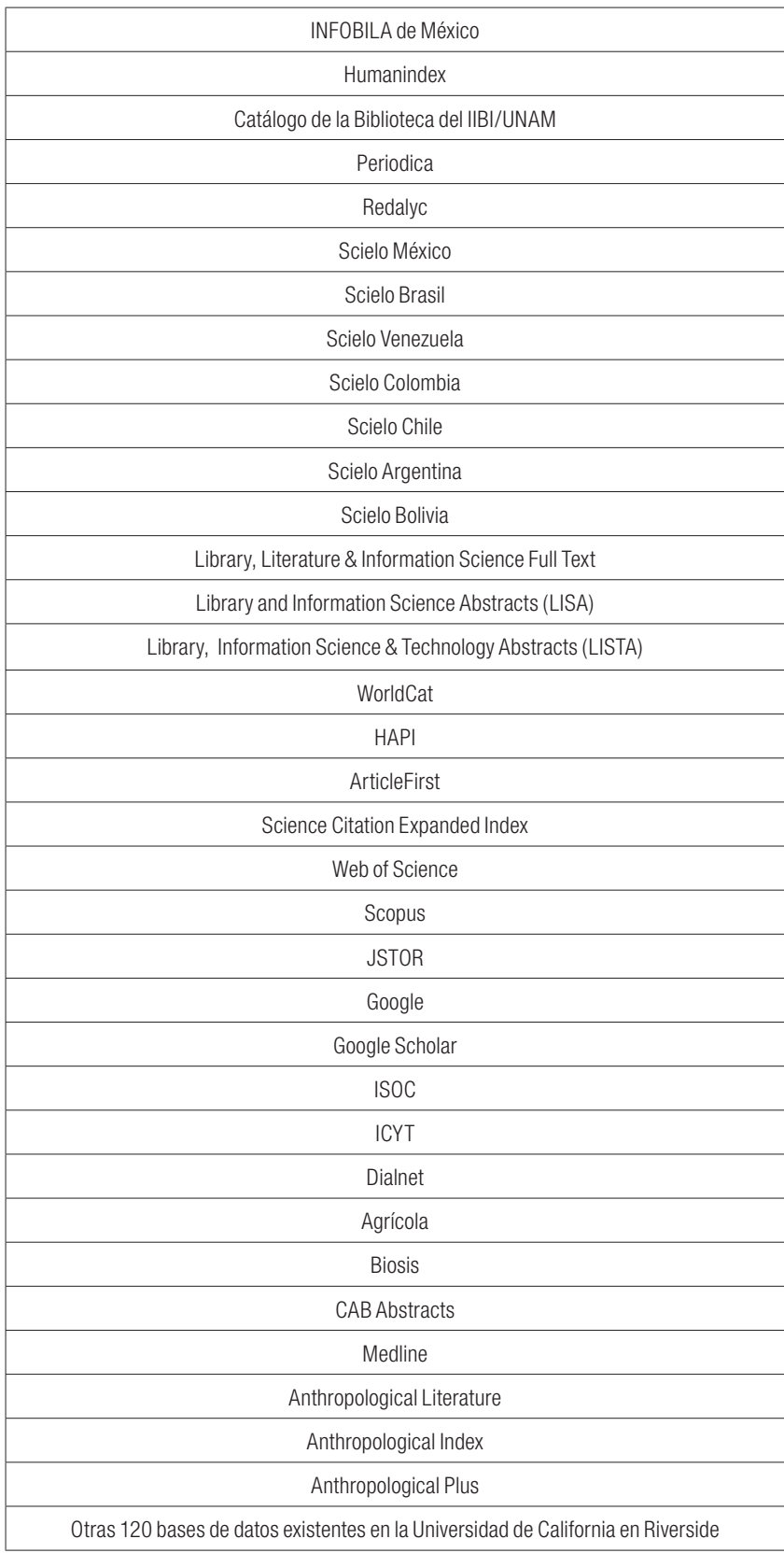


Para citar este texto:

Restrepo Arango, Cristina y Rubén Urbizagástegui Alvarado. 2017. "Red de co-palabras en la bibliometría mexicana". Investigación Bibliotecológica: archivonomía, bibliotecología e información 73 (31): 17-45.

http://dx.doi.org/10.22201/iibi.24488321xe.2017.73.57845 